\title{
Analysis and Design of Amplifying Circuit Resisting Temperature Disturbance of the Food Material Electric Heating Equipment
}

\author{
Minghui You ${ }^{1}$, Xinyu Yu ${ }^{2}$, Qixiang Sun ${ }^{1}$, Liping Yin ${ }^{1}$,Shijun $\mathrm{LI}^{1}$, \\ Xue $\mathrm{Li}^{1}$, Jingsheng $\mathrm{Liu}^{1, \text { a }}$ \\ ${ }^{1}$ Jilin Agricultural University, Changchun 130118, Jilin, China \\ ${ }^{2}$ Jilin Institute of agricultural machinery, Changchun 130021, Jilin, China \\ amhyou000@163.com
}

Keywords: heating equipment, amplifier circuit, the static operating point

\begin{abstract}
Food material heating equipment need to resist temperature interference amplifier circuit. Although the collector current has increased when the ambient temperature rises, but the partial amplifier circuit to make adjustments quickly to suppress the increase in the trend, making the collector current is basically the same, to ensure that the amplifier circuit to stabilize the work and almost independent of temperature.
\end{abstract}

\section{Introduction}

Some food materials, heating equipment, fixed bias selection amplifier circuit [1]. This circuit had the advantages of simple structure and convenient debugging[2]. However, this circuit there was a drawback: that was when the ambient temperature changes, would cause a static point of instability, and sometimes the circuit didn't work[3]. In order to solve this problem, the paper chose an amplification circuit to resist temperature interference[4]. There were many factors that affect the static operating point: for example, fluctuations in power supply voltage, component aging, and temperature on the transistor characteristics of the parameters[5]. We chose the high stability of the power supply and high-quality components to be overcome, however, the third factor of the temperature on the transistor characteristics of the parameters, cannot be avoided.

\section{Overall System Framework}

\section{Heating block diagram}

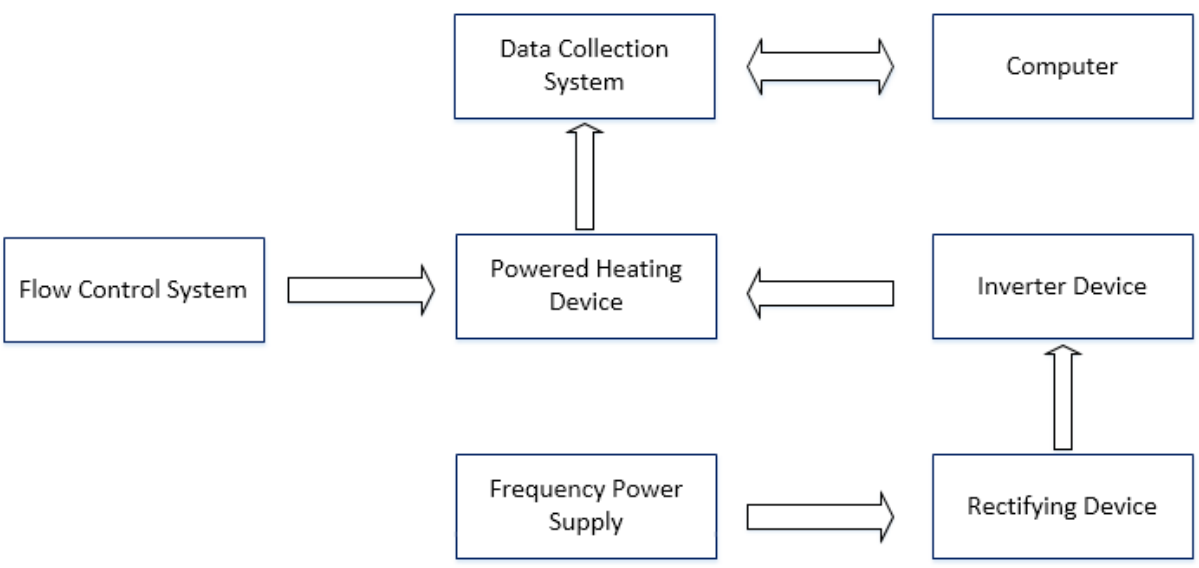

Fig1. Schematic diagram of continuous energization heating system 


\section{Operating Principle}

\subsection{Working Conditions}

Partial bias amplifier circuit in order to stabilize the work meeted the following two con ditions:

$$
\begin{gathered}
I_{2} \gg I_{B} \\
V_{B} \gg U_{B E}
\end{gathered}
$$

The DC path of the partial-bias amplifier circuit was very simple. For the DC component, the capacitor was equivalent to an open circuit, so that the DC path of the partial-bias amplifier circuit was composed of two loops: The first loop, the base of the transistor, emitter resistance, to the ground, that was, back to the DC power supply negative.The second loop, DC power, collector resistance, the collector of the transistor , emitter resistance to ground[6].

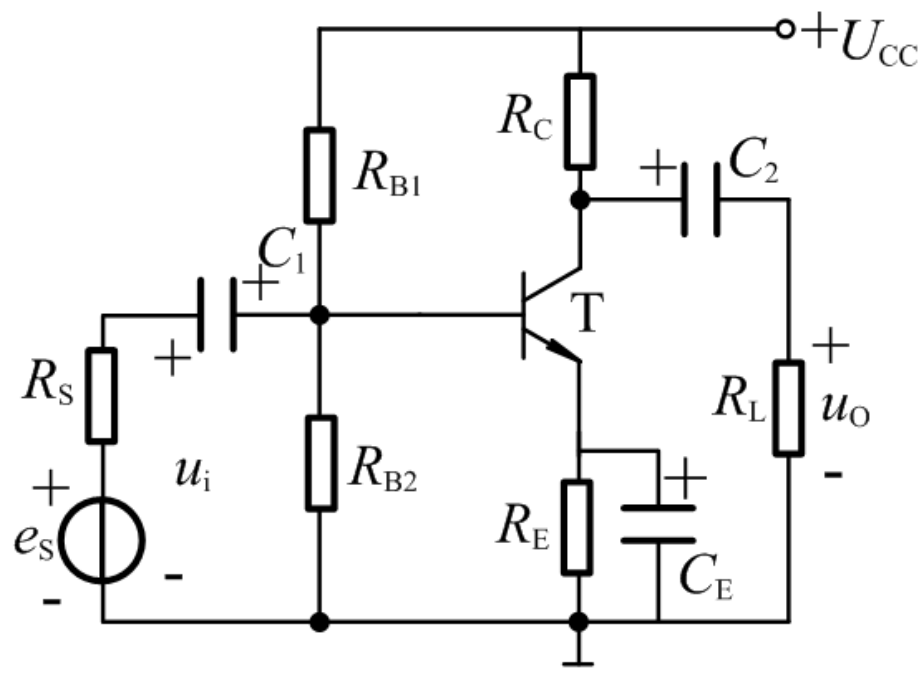

Fig1.Amplified circuit diagram

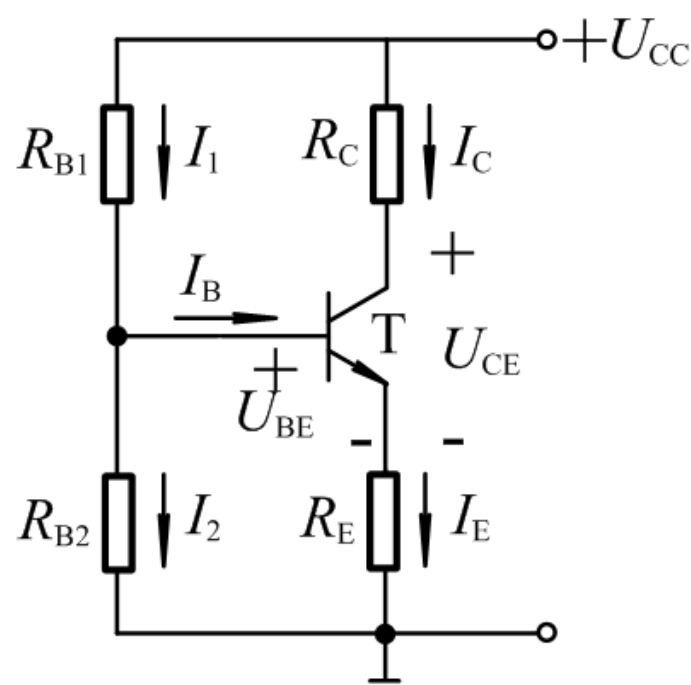

Fig2. Constant current diagram 
In the DC path, according to the Kirchhoff current law, can be $I_{1}=I_{2}+I_{B}$. When $I_{2}>I_{B}$, we approximated that $I_{1}$ and $I_{2}$ were equal, since the current flowing through the resistors $R_{B 1}$ and $R_{B 2}$ were approximately equal, we approximated that the resistance $R_{B 1}$ and $R_{B 2}$ were in series. Then the base potential, equal to the resistance $R_{B 2}$ on the power supply voltage partial pressure value.

Then analyze the second condition: $V_{B} \gg U_{B E}$. Type the formula here. Current return to the DC path, the emission junction voltage drop, equal to the base potential $V_{B}$ minus the emitter potential $V_{E}$, and $V_{E}$ is equal to the resistance $R_{E}$ and current $I_{E}$ product. The emitter current $I_{E}$ equal to the collector current $I_{C}$.

From the above analysis we saw, for the partial bias amplifier circuit when these two conditions were satisfied: the collector current $I_{C}$ only from the base potential $V_{B}$ and emitter resistance $R_{E}$ decision. And the base potential $V_{B}$ was only related to the base resistance and the supply voltage $V_{C C}$. Therefore, the collector current $I_{C}$ only by the resistance and power, with the impact of the static operating point of the transistor characteristics of the stable parameters. So this circuit can be stable, which didn't affected by temperature[7].

3.2 Work Process

In order to understood the working characteristics of this circuit, we studied it stable working point of static work process.

Assuming that the ambient temperature of the circuit was increased, leading to an increase in the collector current $I_{C}, I_{C}$ would be approximately equal to the emitter current $I_{E}$ will increase . The emitter current $I_{E}$ increases in the emitter resistance of the case didn't changed the emitter potential $V_{E}$ necessarily increased. So, $V_{E}$ in turn would bring to the whole circuit to change it. Through the front of the learning we knew, emitter potential $V_{E}$ was equal to the base potential $V_{B}$ minus the emitter junction voltage drop $U_{B E}$, and the base potential by the base resistance supplied voltage decision. Set a partial bias amplifier circuit, its base potential was fixed. So $V_{E}$ increased, $V_{B}$ unchanged, $U_{B E}$ was bound to decrease. By the input characteristics of the transistor we knew that the base current $I_{B}$ would decrease as the $U_{B E}$ decreases. If an amplifier circuit was given, its voltage amplification factor $\beta$ was known, and $I_{C}=\beta I_{B}$, then, $I_{B}$ was reduced, $I_{C}$ was automatically reduced down.

\section{Summary}

In this paper, the temperature of the working point of the first analysis of the circuit voltage divider amplifier circuit for static analysised and dynamic analysised. When the ambient temperature increases, although the collector current increases, the partial bias amplifier circuit to make adjustments quickly to suppress the increase in the trend, making the collector current was basically the same, to ensure that the The amplifier circuit can work stably, almost independent of temperature.

\section{Acknowledgments}

The authors wish to express their gratitude to the projects: Jilin Province Education Department Project (No. 2015Y174) , Jilin Agricultural University learning program , Jilin Provincial 
Department of human resources and social security Project (No. 2015Y24)for their generous support of this work.

\section{References}

[1]Zuo Quansheng. A study on the appropriate stable bias point for transistor amplifiers [J]Journal of Changzhou Institute of Technology,2009,22(3):31-33.

[2]Ren Xiufang.A discussion on quiescent point in analog electronic technique course. [J]Journal of EEE,2013,35(2):69-73.

[3]Li Yongqing.Multisim simulation of partial pressure and self-bias pressure common source amplification circuit. [J]Modern Electronics Technique,2011,34(13):189-191.

[4]Dong hua.A comparative study of Chinese and foreign textbooks on DC analysis for four-resistor bias common-emitter circuit. [J]Journal of Nanchang Hangkong University:2015,29(2):104-108.

[5] Lian Hanli, Theory analysis on volt-ammetry characteristic curves of diode [J] Journal of Xi'An University of Post and Telecommunication, 2008.13(5):150-152.

[6] Wang Wenting,Diode Volt-ampere Characteristic Automatic Test System. [J]Instrument Technique and Sensor,2015.11:65-66.

[7] Ren Junyuan.Influence of quiescent points stability on dynamic performance of emitter follower. [J] Modern Electronics Technique,2013,1(1):76-78. 